\title{
Programação por Metas, Análise por Envoltória de Dados e Teoria Fuzzy na avaliação da eficiência sob incerteza: Aplicação em minifábricas do segmento de autopeças
}

\author{
Goal Programming and Data Envelopment Analysis combined with \\ Fuzzy Theory to evaluate efficiency under uncertainty: Application in \\ mini-factories of the auto parts segment
}

\author{
Aneirson Francisco da Silva ${ }^{1}$ \\ Fernando Augusto Silva Marins ${ }^{1}$ \\ Marcos Vinícius Brandão Santos ${ }^{1}$
}

\begin{abstract}
Resumo: O mercado mundial de peças para automóveis é tipicamente caracterizado pela presença marcante de fornecedores globais que são continuamente pressionados a reduzir custos e aumentar a produtividade e a competitividade. Nesse contexto, este artigo propõe novos modelos, denominados FGPDEA, que combinam a Análise por Envoltória de Dados (Data Envelopment Analysis - DEA), a Teoria Fuzzy e a Programação por Metas (Goal Programming - GP), visando aumentar a discriminação entre as Unidades Tomadoras de Decisão (Decision Making Units - DMUs) num ambiente sob incerteza. Foi feita uma aplicação real dos modelos FGPDEA para se avaliar a eficiência de sete minifábricas do segmento de autopeças. Os resultados obtidos mostraram-se aderentes à realidade estudada, identificando de forma confiável quais minifábricas são eficientes e quais delas são mais sensíveis ao efeito da incerteza.
\end{abstract}

Palavras-chave: Eficiência. Programação por Metas. Análise por Envoltória de Dados. Teoria Fuzzy. Segmento de Autopeças.

\begin{abstract}
The global market for automotive parts is typically characterized by the strong presence of global suppliers, which are continually pressured to reduce costs, and increase productivity and competitiveness. In this context, this paper describes a new FGPDEA model that combines Data Envelopment Analysis (DEA) and Fuzzy Goal Programming, thereby aiming to increase the discrimination among Decision Making Units (DMUs) in an environment under uncertainty. A real application of the FGPDEA model was carried out to evaluate the efficiency of seven mini-factories (DMUs) of the auto parts segment. The results obtained in this real problem were adherent to the reality studied, reliably identifying which mini-factories were efficient and which ones were more sensitive to the effect of uncertainty.
\end{abstract}

Keywords: Efficiency. Goal Programming. Data Envelopment Analysis. Fuzzy Theory. Auto Parts Segment.

\section{Introdução}

O segmento de autopeças vem enfrentando grandes desafios, pois as montadoras devem buscar continuamente atender padrões cada vez mais altos de qualidade mantendo preços competitivos. Segundo Cook e Seiford (2009), procedimentos para medir eficiência são um assunto de interesse para as organizações produtivas. Nesse cenário, as técnicas de Pesquisa Operacional (PO), como a Análise por Envoltória de Dados (Data Envelopment Analysis - DEA) proposta por Charnes, Cooper e Rhodes (1978), podem auxiliar na avaliação da

eficiência das iniciativas das empresas no sentido de se destacarem no mercado.

A DEA permite, entre outras funcionalidades, comparar entidades similares, denominadas Unidades Tomadoras de Decisão (Decision Making Units - DMUs), que têm os mesmos recursos (inputs ou entradas) e oferecem produtos (outputs ou saídas) similares, em termos da sua eficiência. Com modelos da DEA, a avaliação da eficiência das DMUs é realizada por meio da identificação de uma fronteira de eficiência (com relações saída/entrada $=100 \%$ ), na

\footnotetext{
Faculdade de Engenharia, Universidade Estadual Paulista - UNESP, Av. Ariberto Pereira da Cunha, 333, CEP 12516-410,

Guaratinguetá,SP, Brasil, e-mail: aneirson@yahoo.com.br; fmarins@feg.unesp.br; brandao_vbs@ hotmail.com
}

Recebido em 9/4/2013 — Aceito em 11/3/2014

Suporte financeiro: CNPq e Capes. 
qual se localizarão as DMUs consideradas eficientes. Assim, numa empresa industrial ou de serviços, a DEA permite que sejam evidenciadas quais são as operações ineficientes e proporciona subsídios aos gestores na difícil atividade de tomada de decisão vinculada à redução dos recursos produtivos e aumentos dos níveis de produção (KAO; LIN, 2012).

Corroborando essas afirmações, para Kao e Liu (2000), medir a eficiência das DMUs em ambientes industriais e de serviços é uma tarefa árdua, pois usualmente estão envolvidas variáveis econômicas complexas, como taxas de juros, tributação, nível de emprego, demanda, dentre outras. Nesse sentido, Figueiredo e Mello (2009) comentam que em cenários complexos torna-se fundamental uma metodologia eficaz e abrangente para medição dos resultados com fins de monitoramento e correções, visando a otimização de processos.

Já Wen, Qin e Kang (2011) comentam que nos modelos clássicos de DEA assume-se que as entradas e saídas são estimadas de forma exata, ou seja, o analista considera que não há erros ou informações subjetivas (incerteza) nesses valores. De acordo com Hatami-Marbini, Emrouznejad e Tavana (2011), as estimações dos valores de entradas e saídas em problemas reais podem ser imprecisas, gerando valores de eficiência com baixa confiabilidade.

Nesse contexto, Wen, Qin e Kang (2011) e Wen e You (2007) também comentam que as DMUs podem ser classificadas em duas classes, eficientes e ineficientes, e que a incorporação da incerteza como um erro de medição deveria ser inserida nas entradas e saídas de modo a tornar o cálculo das eficiências das DMUs mais confiáveis do ponto de vista prático. Conforme Cooper, Seiford e Tone (2006), as variáveis de entrada e de saída para cada DMU devem atender alguns critérios como:

- As variáveis e DMUs devem ser escolhidas de modo a representarem o interesse dos gestores;

- Há dados numéricos positivos para as variáveis de entrada e de saída, sendo que se deve preferir um uso menor do número de variáveis de entradas comparado ao múmero das variáveis de saídas;

- Recomenda-se aplicar os modelos clássicos CCR e BCC quando o número de DMUs é igual ou maior a três vezes a soma do número de variáveis de entrada e de saída.

Quando o terceiro critério não é satisfeito, os modelos clássicos de DEA - CCR (Retorno Constante de Escala), de Charnes, Cooper e Rhodes (1978), e BCC (Retorno Variável de Escala), de Banker, Charnes e Cooper (1984) - não proporcionam uma boa discriminação das DMUs quanto à eficiência.

Em estudos recentes têm-se estudado a combinação da lógica Fuzzy com os modelos DEA para tentar tratar o efeito da incerteza nas entradas e saídas, tornando o cálculo das eficiências mais confiável. Nessa linha pode-se citar: Kao e Lin (2000, 2012), Hatami-Marbini, Emrouznejad e Tavana (2011), Wen, Qin e Kang (2011), Wen e You (2007), Lertworasirikul et al. (2003), Entani, Maeda e Tanaka (2002), Cooper et al. (1999), e Guo e Tanaka (2001).

Numa linha de pesquisa recente, Bal, Örkcü e Çelebioğlu (2010) propuseram modelos conhecidos como GPDEA agregando os conceitos da Programação por Metas (Goal Programming - GP), proposta por Charnes e Cooper (1961), nos modelos DEA, para melhorar a sua capacidade de discriminação das DMUs. Porém cabe mencionar que os modelos GPDEA não consideram a ocorrência da incerteza na análise da eficiência, dessa forma, uma das contribuições deste trabalho é a combinação da lógica Fuzzy com o modelo GPDEA. Para detalhes sobre aplicações de modelos de Programação Por Metas recomenda-se a leitura do trabalho de Silva, Marins e Montevechi (2013a).

Este trabalho teve como objetivo geral propor extensões dos modelos GPDEA que possam tratar a ocorrência de incerteza que usualmente está presente em aplicações reais e, para tanto, foram incorporados nos modelos GPDEA alguns elementos da Teoria Fuzzy (ZADEH, 1973, 1978). Esses modelos são aqui denominados modelos FGPDEA-CCR (Fuzzy Goal Programming and Data Envelopment Analysis - FGPDEA-CCR) e FGPDEA-BCC (Fuzzy Goal Programming and Data Envelopment Analysis - FGPDEA-BCC) e, como objetivo específico neste trabalho, foram aplicados para avaliar a eficiência de sete minifábricas de uma empresa do segmento de autopeças num cenário sob incerteza, o que permitiu a validação dos modelos propostos.

Este artigo está assim estruturado: na seção 2 estão conceitos e modelos da Programação por Metas, da DEA e da Teoria Fuzzy que foram úteis no desenvolvimento dos modelos aqui propostos. $\mathrm{Na}$ seção 3 está a descrição do problema e do método de pesquisa adotado. Na seção seguinte estão os modelos FGPDEA-CCR e FGPDEA-BCC propostos, aplicados na determinação da eficiência de minifábricas do setor de autopeças, assim como os resultados obtidos e sua análise. Finalmente, na seção 5 estão as considerações finais do trabalho, seguidas das referências bibliográficas.

\section{Modelos GPDEA e modelos Fuzzy DEA}

Nesta seção são apresentados os modelos da DEA, da GP e da Teoria Fuzzy que foram úteis para a formulação dos modelos FGPDEA-CCR e FGPDEA-BCC que são o foco deste trabalho. 
A DEA permite, entre outros aspectos relevantes, a identificação das DMUs que sejam referência (benchmarking) para as demais analisadas. A sua origem se deve a Charnes, Cooper e Rhodes (1978), que desenvolveram um modelo a fim de se obter uma nova medida de eficiência para avaliar programas públicos. Os pesos para variáveis de entrada e saída do modelo geral da DEA podem ser obtidos a partir da solução do modelo proposto por Charnes, Cooper e Rhodes (1978), dado pelas Equações 1 a 4:

$$
\max E_{j}=\sum_{r=1}^{s} u_{r} \cdot y_{r 0} / \sum_{i=1}^{m} v_{i} \cdot x_{i 0}
$$

s.a.:

$$
\begin{gathered}
\left(\sum_{r=1}^{s} u_{r} y_{r j} / \sum_{i=1}^{m} v_{i} x_{i j}\right) \leq 1, j=1,2, \ldots, n \\
u_{r} \geq 0, \quad r=1,2, \ldots, s \\
v_{i} \geq 0, \quad i=1,2, \ldots, m
\end{gathered}
$$

sendo a $\mathrm{DMU}_{0}$ a DMU sob avaliação ou análise; $w_{o}$ a eficiência relativa da $\mathrm{DMU}_{0} ; y_{r 0}$ e $x_{i o}$ os dados de saídas e entradas para a $\mathrm{DMU}_{0} ; j$ o índice da DMU, $j=1 \ldots$, $n ; r$ o índice da saída, $\operatorname{com} r=1, \ldots, s ; i$ o índice da entrada, $i=1, \ldots, m ; y_{r j}$ o valor da $r$-ésima saída para a $j$-ésima DMU; $x_{i j}$ o valor da $i$-ésima entrada para a j-ésima DMU; $u_{r}$ o peso associado à $r$-ésima saída; e $v_{i}$ o peso associado à $i$-ésima entrada. Observe-se que: se $E_{j}=1$, a $\mathrm{DMU}_{0}$ é eficiente quando comparada às demais unidades consideradas no modelo e se $0 \leq E_{j}<1$, a DMU$_{0}$ é ineficiente.

A abordagem da GP original considera que os gestores devem associar metas (valores alvos ou goals) aos objetivos $f_{i}(x)$ e decidir qual é a penalização mais adequada para as variáveis de desvio associadas à obtenção de valores acima $\left(d^{+}\right)$ou abaixo $\left(d_{i}^{-}\right)$do valor alvo estabelecido. Uma aplicação interessante da GP foi idealizada por Bal, Örkcü e Çelebioğlu (2010), criando a GPDEA (Goal Programming and Data Envelopment Analysis - GPDEA), no sentido de melhorar o desempenho de modelos DEA na tarefa de melhor discriminar as DMUs eficientes. Os modelos GPDEA evitam a ponderação excessiva que ocorre quando se aplicam os modelos clássicos da DEA em cenários onde eles têm baixo poder de discriminação das DMUs. O modelo GPDEA de Bal, Örkcü e Çelebioğlu (2010) é derivado de um modelo DEA multiobjetivo proposto por Li e Reeves (1999) e descrito pelas expressões 5 a 11 :

$$
\begin{aligned}
& \min d_{0}\left(\text { or } \max \sum_{r=1}^{s} u_{r} \cdot y_{r 0}\right) \\
& \min M \\
& \min \sum_{j=1}^{n} d_{j}
\end{aligned}
$$

$$
\sum_{i=1}^{m} v_{i} x_{i o}=1
$$

s.a.:

$$
\begin{gathered}
\sum_{r=1}^{s} u_{r} y_{r j}-\sum_{i=1}^{m} v_{i} x_{i j}+d_{j}=0, \quad j=1,2, \ldots, n \\
M-d_{j} \geq 0, \quad j=1,2, \ldots, n \\
u_{r} \geq 0, \quad r=1,2, \ldots, s \\
v_{i} \geq 0, \quad i=1,2, \ldots, m \\
d_{j} \geq 0, \quad j=1,2, \ldots, n
\end{gathered}
$$

sendo $\mathrm{d}_{j}$ a variável de desvio para a DMU $j ; \mathrm{M}=\max$ $\left\{d_{j}\right\}$ o valor máximo de variação das variáveis de desvio; $d_{0}$ o valor da eficiência para a DMU que está sendo analisada. Os demais parâmetros e variáveis já foram definidos anteriormente.

Bal, Örkcü e Çelebioğlu (2010) associaram metas às três funções objetivos do modelo 5 a $11 \mathrm{e}$ desenvolveram os modelos GPDEA-CCR (Retorno Constante de Escala) e GPDEA-BCC (Retorno Variável de Escala) que estão expressos, respectivamente, por 12 a 20 e 21 a 29 :

$$
\min a=\left(d_{1}^{-}+d_{1}^{+}+d_{2}^{-}+\sum_{j=1}^{n} d_{3 j}^{-}+\sum_{j=1}^{n} d_{j}\right)
$$

s.a.:

$$
\begin{gathered}
\sum_{i=1}^{m} v_{i} x_{i o}+d_{1}^{-}-d_{1}^{+}=1 \\
\sum_{r=1}^{s} u_{r} y_{r 0}+d_{2}^{-}-d_{2}^{+}=1 \\
\sum_{r=1}^{s} u_{r} y_{r j}-\sum_{i=1}^{m} v_{i} x_{i j}+d_{j}=0, \quad j=1,2, \ldots, n \\
M-d_{j}+d_{3 j}^{-}-d_{3 j}^{+}=0, \quad j=1,2, \ldots, n \\
u_{r} \geq 0, \quad r=1,2, \ldots, s \\
v_{i} \geq 0, \quad i=1,2, \ldots, m \\
d_{j} \geq 0, \quad j=1,2, \ldots, n \\
d_{1}^{+}, d_{1}^{-}, d_{2}^{+}, d_{2}^{-} \geq 0, \quad d_{3 j}^{-}, d_{3 j}^{+} \geq 0
\end{gathered}
$$




$$
\min a=\left(d_{1}^{-}+d_{1}^{+}+d_{2}^{-}+\sum_{j=1}^{n} d_{3 j}^{-}+\sum_{j=1}^{n} d_{j}\right)
$$

s.a.:

$$
\begin{gathered}
\sum_{i=1}^{m} v_{i} x_{i o}+d_{1}^{-}-d_{1}^{+}=1 \\
\sum_{r=1}^{s} u_{r} y_{r 0}+c_{0}+d_{2}^{-}-d_{2}^{+}=1 \\
\sum_{r=1}^{s} u_{r} y_{r j}-\sum_{i=1}^{m} v_{i} x_{i j}+c_{0}+d_{j}=0 \quad j=1,2, \ldots, n \\
M-d_{j}+d_{3 j}^{-}-d_{3 j}^{+}=0, \quad j=1,2, \ldots, n \\
u_{r} \geq 0, \quad r=1,2, \ldots, s \\
v_{i} \geq 0, \quad i=1,2, \ldots, m \\
d_{j} \geq 0, \quad j=1,2, \ldots, n \\
d_{1}^{+}, d_{1}^{-}, d_{2}^{+}, d_{2}^{-} \geq 0, \quad d_{3 j}^{-}, d_{3 j}^{+} \geq 0
\end{gathered}
$$

sendo $d_{1}, d^{+}{ }_{1}$ as variáveis de desvio indesejáveis para a meta que restringe a soma ponderada das entradas ao valor de $1(100 \%) ; d^{+}{ }_{2}$ a variável de desvio indesejável para a meta que restringe a soma ponderada das saídas a ser menor ou igual a 1 (100\%); $d_{2}{ }_{2}$ a variável de desvio desejável para a meta que restringe a soma ponderada das saídas a ser menor ou igual a $1(100 \%) ; d_{3 j}^{-}$a variável de desvio indesejável, da DMU $j$, para a meta que tem M como o desvio máximo; $d^{+}{ }_{3 j}$ as variáveis de desvio desejável, da DMU $j$, para a meta que tem M como o desvio máximo; $d_{j}$ a variável de desvio indesejável, da $\mathrm{DMU} j$, com relação ao desvio máximo M que é o desvio máximo; e c $\mathrm{c}_{0}$ a variável irrestrita de sinal que determina o retorno variável de escala. Cabe mencionar, que os modelos GPDEA são modelados pela função Weighted Goal Programming- WGP (CHARNES; COOPER, 1961, 1962).

A Teoria Fuzzy vem sendo usada como técnica para modelar a incerteza nos modelos DEA, tendo como base os modelos da Programação Linear Fuzzy. Nessa linha, conhecida como DEA Fuzzy, Lertworasirikul et al. (2003) estabeleceram o modelo DEA-CCR com coeficientes Fuzzy (modelo FCCR), que pode ser expresso por 30 a 34 :

$$
\operatorname{Max} \tilde{E}_{j}=\sum_{r=1}^{s} u_{r} \tilde{y}_{r 0}
$$

s.a.:

$$
\sum_{i=1}^{m} \tilde{x}_{i 0} v_{i}=1
$$

$$
\begin{gathered}
\sum_{r=1}^{s} u_{r \tilde{Y}_{r j}}-\sum_{i=1}^{m} v_{i} \tilde{X}_{i j} \leq 0, \quad j=1,2, \ldots, n \\
u_{r} \geq 0, \quad r=1,2, \ldots, s \\
v_{i} \geq 0, \quad i=1,2, \ldots, m
\end{gathered}
$$

sendo $\mathrm{DMU}_{0}$ a DMU que está sob análise, $\tilde{x}_{i 0}$ as variáveis Fuzzy do $i$-ésima entrada da $\mathrm{DMU}_{0} ; \tilde{y}_{\mathcal{X}^{0}}$ as variáveis $F u z z y$ da $r$-ésima saída da $\mathrm{DMU}_{0} ; \widetilde{X}_{i j}$ a matriz das variáveis $F u z z y$ da $i$-ésima entrada da $\operatorname{DMU} j ; \tilde{Y}_{r j}$ a matriz das variáveis Fuzzy da $r$-ésima saída DMU $j$.

Para se obter a formulação do Modelo BCC basta inserir de forma aditiva a variável auxiliar irrestrita $c_{o}$ (também conhecida como fator de escala) na função objetivo 30 e na restrição 31 . Ainda segundo Lertworasirikul et al. (2003), diferentemente do que ocorre nos modelos DEA tradicionais, o valor da função objetivo 30 poderá ter um valor acima de 1, devido às restrições Fuzzy (GARCIA; MELO; SCHIRRU, 2009). Hatami-Marbini, Emrouznejad e Tavana (2011) elencaram as principais abordagens para tratar os modelos Fuzzy DEA:

- Abordagem baseada em tolerâncias (The tolerance approach);

- Abordagem baseada em nível- $\alpha$ (The $\alpha$-level based approach);

- Abordagem baseada em ranqueamento Fuzzy (The Fuzzy ranking approach);

- Abordagem baseada em possibilidades (The possibility approach).

Neste artigo foi adotada a abordagem baseada no nível- $\alpha$ que, conforme Hatami-Marbini, Emrouznejad e Tavana (2011), é o modelo mais popular de Fuzzy DEA, com muitas publicações associadas (KAO; LIU, 2000). O valor de $\alpha \in[0,1]$ permite a geração de cenários, ou seja, diferentes valores de eficiência, respeitando-se a faixa de variação determinada pela função de pertinência. Nesses modelos, têm-se $\tilde{X}_{i j}$ e $\widetilde{Y}_{r j}$ - que são, respectivamente, os parâmetros Fuzzy da $i$-ésima entrada e $r$-ésima saída da DMU $j$, eles são aproximadamente conhecidos e podem ser representados por conjuntos Fuzzy, por meio de funções de pertinência $\mu \tilde{X}_{i j}$ e $\mu \tilde{Y}_{r j^{\prime}}$. Dessa forma, os modelos Fuzzy DEA podem ser formulados utilizando-se $\mathrm{S}\left(\widetilde{X}_{i j}\right)$ e $\mathrm{S}\left(\widetilde{Y}_{r j}\right)$ - que são os valores desses parâmetros para um determinado cenário $S$. Com os $\alpha$-cortes (nível- $\alpha$ ) que geram um conjunto de cenários para $\widetilde{Y}_{r j}$ e $\widetilde{X}_{i j}$, têm-se, conforme definido em Kao e Liu (2000) $(35,36)$ : 


$$
\begin{aligned}
& \left(X_{i j}\right) \alpha=\left\{x_{i j} \in S\left(\tilde{X}_{i j}\right) \mid \mu_{\tilde{X}_{i j}}\left(x_{i j}\right) \geq \alpha\right\}, \quad \forall i, j, \\
& \left(Y_{r j}\right) \alpha=\left\{y_{r j} \in S\left(\tilde{Y}_{r j}\right) \mid \mu_{\tilde{Y}_{r j}}\left(y_{r j}\right) \geq \alpha\right\}, \quad \forall r, j
\end{aligned}
$$

A DEA Fuzzy pode ser transformada em uma família de modelos DEA com diferentes níveis de incerteza: $\left\{\left(X_{i j}\right) \alpha \mid 0 \leq \alpha \leq 1\right\}$ e $\left\{\left(Y_{r j}\right) \alpha \mid 0 \leq \alpha \leq 1\right\}$. Os resultados de cada cenário identificam a faixa de variação da incerteza nos dados de saída e entrada do modelo DEA (KAO; LIU, 2000; KAUFMANN, 1975; YAGER, 1986; ZADEH, 1973, 1978), sendo o nível- $\alpha$ definido pelas Equações 37 e 38, conforme Kao e Liu (2000):

$$
\begin{gathered}
\left(X_{i j}\right) \alpha=\left[\left\{\min _{x_{j}} x_{i_{j}} \in S\left(\tilde{X}_{i j}\right) \mid \mu_{X_{i j}}\left(x_{i j}\right) \geq \alpha\right\}, \max _{x_{j}}\left\{x_{i j} \in S\left(\tilde{X}_{i j}\right) \mid \mu_{X_{i j}}\left(x_{i j}\right) \geq \alpha\right\}\right] \\
\left(Y_{r j}\right) \alpha=\left[\left\{\min _{y_{j}} y_{r j} \in S\left(\tilde{Y}_{r j}\right) \mid \mu_{\tilde{Y}_{j}}\left(y_{r j}\right) \geq \alpha\right\}, \max _{y_{i j}}\left\{y_{r j} \in S\left(\tilde{Y}_{r j}\right) \mid \mu_{\tilde{Y}_{j}}\left(y_{r j}\right) \geq \alpha\right\}\right]
\end{gathered}
$$

Kao e Liu (2000), baseando-se em Zadeh (1973, 1974) e Zimmermann (1991), estabeleceram que a função de pertinência que define a eficiência da DMU $j$ pode ser expressa por (39):

$$
\mu_{\tilde{E}_{j}}(z)=\sup _{x, y} \min =\left\{\mu_{\tilde{X}_{i j}}\left(x_{i j}\right), \mu_{\tilde{r}_{r j}}\left(y_{r j}\right), \forall i, r, j \mid z=E_{j}(x, y)\right\}
$$

sendo $E_{j}(x, y)$ obtido por 1 a 4 . A a a $\tilde{E}_{\text {bordagem para }}$ construção da função de pertinência $\mu \tilde{E}_{j}$ proposta neste trabalho adotou o nível- $\alpha$ de $\mu \widetilde{E}_{j}$, sendo o valor da eficiência em cada cenário $\tilde{E}_{j}$ obtido por 30 a 34 . Para detalhes recomenda-se a leitura dos trabalhos de Kao e Liu (2000, 2012) e Hatami-Marbini, Emrouznejad e Tavana (2011).
A Figura 1 contempla geometricamente a posição dos parâmetros dos modelos DEA modelados pela função de Pertinência Triangular. Sendo $\tilde{x}_{i 0}$ ou $\tilde{x}_{i j}$ e $\tilde{y}_{r 0}$ ou $\tilde{y}_{r j}$ correspondentes ao limite inferior de variação associado aos inputs e outputs das DMUs. Já $\tilde{X}_{i 0}$ ou $\tilde{X}_{i j}$ e $\tilde{Y}_{r 0}$ ou $\tilde{Y}_{r j}$ correspondem ao limite superior de variação dos inputs e outputs das DMUs. Destaque-se que o valor da média da função de Pertinência Triangular está associado aos valores desses parâmetros num cenário sem incerteza. Na Figura 1, no eixo das ordenadas, têm-se os níveis de pertinência e, no eixo das abscissas, estão os valores vinculados ao input analisado.

Pelo modelo proposto por Kao e Liu (2000), dois níveis de eficiência são gerados, os quais estão associados ao cenário otimista e ao cenário pessimista, sendo que, para o gestor (decisor), o interessante seria dispor de um ranqueamento envolvendo esses níveis. Chen e Klein (1997) contribuíram para esse intento ao proporem um índice $\left(I_{j}\right)$ combinando os dois níveis (do cenário otimista e do cenário pessimista) para cada DMU, conforme 40:

$$
I_{j}=\sum_{p=0}^{g}\left(\left(E_{j p}\right)-c p\right) /\left[\sum_{p=0}^{g}\left(\left(E 2_{j p}\right)-c p\right)-\sum_{p=0}^{g}\left(\left(E_{j}\right)-d p\right)\right], \quad g \rightarrow \infty
$$

sendo $p$ a quantidade de níveis- $\alpha$ (cenários) estabelecidos pelo decisor; $E_{j p}$ o índice de eficiência da DMU $j$ para o cenário pessimista $p ; E 2$ o o índice de eficiência da DMU $j$ para o cenário otimista $p$; $c p=\min _{j p}\left\{E_{j p}\right\}$ o valor mínimo da eficiência dos cenários pessimistas e $d p=\max _{j p}\left\{E 2_{j p}\right\}$ o valor máximo da eficiência dos cenários otimistas.

Observe-se que um valor mais alto de $I_{j}$ está associado a um melhor ranqueamento da DMU $j$



Figura 1. Limite de variação com a incorporação da incerteza. 
em termos da sua eficiência relativa. Conforme 40, o valor de $I_{j}$ é obtido quando o número de nível- $\alpha$ tende ao infinito, mas Chen e Klein (1997) comentam que valores de $p=3$ ou $p=4$ já seriam suficientes para melhorar a discriminação entre as DMUs.

A próxima seção contempla a descrição do problema, os materiais e métodos utilizados.

\section{Descrição do problema e materiais e métodos}

A empresa objeto desta aplicação real é uma fornecedora mundial de autopeças que passou por consecutivas experiências de fusões e aquisições e possui hoje 176 fábricas localizadas em diversos países, sendo oito delas recentemente adquiridas. Este trabalho visou avaliar a eficiência de sete minifábricas de uma unidade fabril dessa empresa localizada na América Latina, considerando a ocorrência de incerteza nos dados. Para tal avaliação foram desenvolvidos os modelos FGPDEA descritos na seção 4. As etapas da pesquisa estão indicadas na Figura 2 e descritas a seguir:

Etapa (a) - Identificação do problema - De comum acordo com os gestores da empresa de autopeças, o problema foi delimitado como identificar a eficiência de sete minifábricas do segmento de autopeças da unidade fabril específica.

Etapa (b) - Coleta de dados - Os dados foram adquiridas no sistema de informações utilizado pela empresa por meio de relatórios gerados nas áreas de contabilidade (faturamento), qualidade e de recursos humanos. A pesquisa foi realizada entre dezembro de 2011 e dezembro de 2012. As Tabelas 1 e 2 apresentam os dados de entradas e saídas, sendo:
- DMUs: $\mathrm{MF}_{1}$ - minifábrica $1, \mathrm{MF}_{2}$ - minifábrica $2, \mathrm{MF}_{3}$ - minifábrica $3, \mathrm{MF}_{4}$ - minifábrica 4 , $\mathrm{MF}_{5}$ - minifábrica 5, $\mathrm{MF}_{6}$ - minifábrica $6 \mathrm{e}$ $\mathrm{MF}_{7}$ - minifábrica 7.

- Saídas: $\mathrm{Y}_{1}$ - Produção total de cada minifábrica e $\mathrm{Y}_{2}$. Delivery Performance que se refere à quantidade de produtos efetivamente entregue ao cliente. Os dados com respeito às saídas (outputs) estão na Tabela 7 do Anexo A.

- Entradas: $X_{1}$ - Custo dos produtos; $\mathrm{X}_{2}$ - Rotatividade da mão de obra em cada minifábrica da empresa, obtida pela razão entre o número de colaboradores que foram desligados pela quantidade de total deles; $\mathrm{X}_{3}$ - Número de afastamentos por doenças ocupacionais e acidentes de trabalho; $\mathrm{X}_{4}$ - Reclamações de clientes (tal variável foi incluída como uma entrada, pois afeta a produção); $\mathrm{X}_{5}$ - Refugo de WIP - Work-in-Process, em [\%]. Os dados com respeito às entradas (inputs) estão na Tabela 8 do Anexo A.

Nesse caso, para aplicar os modelos clássicos de DEA (CCR e BCC) seriam necessárias pelo menos 21 DMUs para o atendimento da condição de Cooper, Seiford e Tone ( 2006) relativa ao poder de discriminação das DMUs. Como a condição não foi satisfeita, optou-se por desenvolver modelos da GPDEA para tratar esse problema.

Adicionalmente, optou-se pela utilização de funções de pertinência triangular para inserir a incerteza nos parâmetros de entrada e de saída do modelo DEA, pois, conforme Liang e Wang (1993), elas representam bem a expertise humana em julgar adequadamente o comportamento de variáveis comuns em diversos

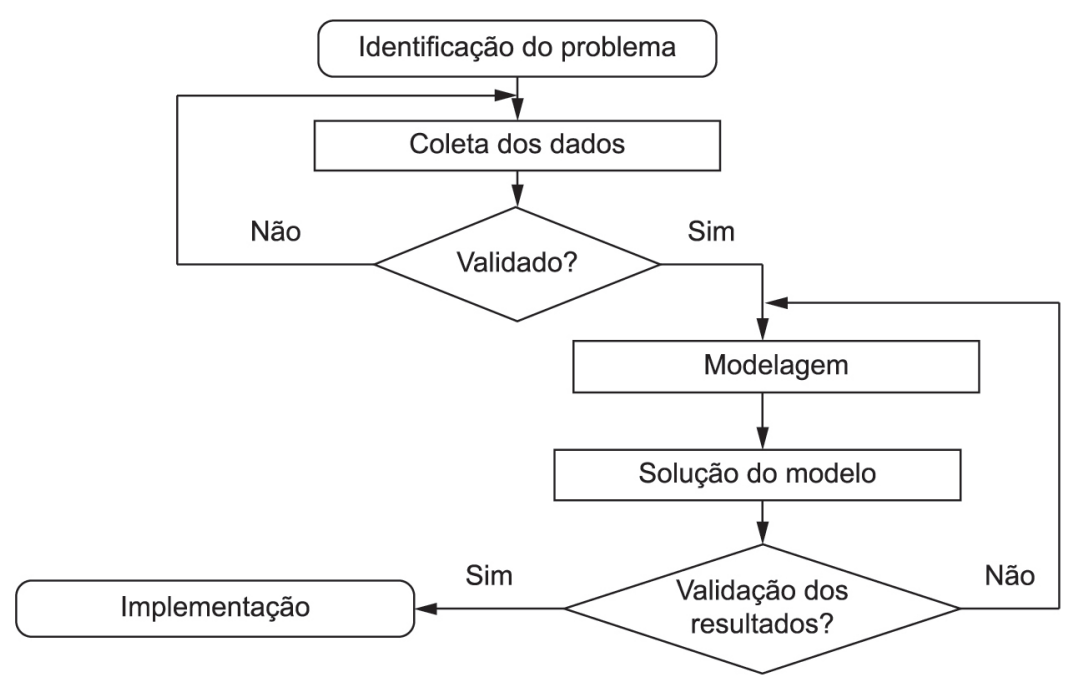

Figura 2. Fases da pesquisa. Fonte: Silva, Marins e Montevechi (2013b). 
tipos de situações práticas. Corroborando esses autores, Aouni, Martel e Hassaine (2009) mostraram diversas aplicações de números triangulares Fuzzy que validam e justificam a adoção de tal método em conjunto com os modelos de GP.

Nesse sentido, os gestores foram consultados sobre as características das variáveis envolvidas e sugeriram as características descritas abaixo para cada uma delas: para $\mathrm{Y}_{1}$ e $\mathrm{Y}_{2}$ sugeriram variações de 100; para $\mathrm{X}_{1}$ sugeriram variações de 0,1 ; para $\mathrm{X}_{2}$, a adoção de desvios de 0,4; para $X_{3}$ sugeriram variações de 100; para $\mathrm{X}_{4}$ sugeriram variações de 1; e para $\mathrm{X}_{5}$ sugeriram variações de $1 \%$. As sugestões dos gestores geraram as funções de pertinência triangulares para as variáveis de saída e entrada Fuzzy, conforme Tabelas 1 e 2.

Etapa (c) - Modelagem - Utilizou-se o software GAMS ${ }^{\circledR}$ (GAMS, 2013) na versão 23.6.5 e o solver CPLEX® na versão 12.2.1. A otimização foi feita por um computador com $8 \mathrm{~GB}$ de RAM processador (Core i7) com 1,2 GHz, plataforma Windows 64 bits. O tempo computacional para otimizar 44 cenários foi de aproximadamente 45 minutos.

Etapa (d) - Solução do modelo - Os resultados estão na seção seguinte.

Etapa (e) - Validação - Foi feita com o apoio dos gestores das minifábricas.

Etapa (f) - Implementação - Foge ao escopo deste trabalho, mas os gestores estão propensos a aplicar os modelos GPDEA em outras situações de seu interesse na empresa.

Segundo Bertrand e Fransoo (2002), esta pesquisa pode ser definida como pesquisa aplicada, pois visa

Tabela 1. Funções de pertinência triangulares de saídas Fuzzy das sete minifábricas.

\begin{tabular}{ccc}
\hline DMU & $\mathbf{Y}_{1}$ & $\mathbf{Y}_{2}$ \\
\hline MF-1 & $(2.410,2.510,2.610)$ & $(2.394,2.494,2.594)$ \\
MF-2 & $(431,531,631)$ & $(407,507,607)$ \\
MF-3 & $(492,592,692)$ & $(425,525,625)$ \\
MF-4 & $(64,164,264)$ & $(59,159,259)$ \\
MF-5 & $(24,124,224)$ & $(24,124,224)$ \\
MF-6 & $(1.185,1.285,1.385)$ & $(1.185,1.285,1.385)$ \\
MF-7 & $(388,488,588)$ & $(384,484,584)$ \\
\hline
\end{tabular}

proporcionar melhorias para a literatura existente, tendo objetivo empírico descritivo, pois o modelo desenvolvido descreve de forma adequada relações causais que podem existir na realidade, favorecendo a compreensão de processos reais. A forma de abordar o problema é quantitativa, sendo o método de pesquisa a modelagem. Na seção seguinte estão descritos com detalhes os modelos GPDEA desenvolvidos e aplicados para determinar a eficiência das minifábricas da empresa objeto do estudo.

\section{Modelos Fuzzy GPDEA-CCR e Fuzzy GPDEA-BCC}

Com base nos modelos desenvolvidos por Bal, Örkcü e Çelebioğlu (2010), conforme 12 a 20 e 21 a 29, foram desenvolvidos os modelos Fuzzy GPDEA-CCR, que é descrito pelas expressões 41 a 49, e Fuzzy GPDEA-BCC, que é descrito pelas expressões 50 a 58, estes modelos foram utilizados para aplicação em um problema real de identificação da eficiência de sete minifábricas (DMUs) de uma empresa do segmento de autopeças. Assim, na sequência estão os índices, parâmetros, variáveis auxiliares e de decisão, funções objetivo e restrições dos modelos propostos, considerando-se a $\mathrm{DMU}_{0}$ como sendo a DMU que está sob análise:

\section{Índices}

- $j$ é o índice das DMUs, $j \in J, J=\{1,2, \ldots, 7\}$;

- $r$ é o índice das saídas, com $r \in R, R=\{1,2\}$;

- $i$ é o índice das entradas, $i \in I, I=\{1,2, \ldots, 5\}$.

\section{Parâmetros}

$\tilde{y}_{r 0}$ e $\tilde{x}_{i 0}$ são, respectivamente, os valores dos limitantes inferiores nos intervalos de definição da função de pertinência triangular para a $r$-ésima saída Fuzzy e a $i$-ésima entrada Fuzzy para a $\mathrm{DMU}_{0}$, considerando a média o valor mais provável, sem incerteza.

$\tilde{Y}_{r 0}$ e Já $\tilde{X}_{i 0}$ são, respectivamente, os valores dos limitantes superiores nos intervalos de definição da função de pertinência triangular para a $r$-ésima saída Fuzzy e a $i$-ésima entrada Fuzzy para a $\mathrm{DMU}_{0}$, considerando a média o valor mais provável, sem incerteza.

Tabela 2. Funções de pertinência triangulares de entradas Fuzzy para as sete minifábricas.

\begin{tabular}{cccccc}
\hline DMU & $\mathbf{X}_{1}$ & $\mathbf{X}_{2}$ & $\mathbf{X}_{3}$ & $\mathbf{X}_{4}$ & $\mathbf{X}_{5}$ \\
\hline MF-1 & $(0,06,0,16,0,26)$ & $(0,02,0,42,0,82)$ & 0 & $(1,2,3)$ & $(3,83,4,83,5,83)$ \\
MF-2 & $(0,59,0,69,0,79)$ & $(0,52,0,92,1,32)$ & $(11,111,211)$ & $(1,2,3)$ & $(10,3,11,3,12,3)$ \\
MF-3 & $(0,41,0,51,0,61)$ & $(0,14,0,54,0,94)$ & $(298,398,498)$ & $(3,4,5)$ & $(0,23,1,23,2,23)$ \\
MF-4 & $(2,21,2,31,2,41)$ & $(0,16,0,56,0,96)$ & $(94,194,294)$ & $(1,2,3)$ & $(14,57,15,47,16,57)$ \\
MF-5 & $(3,4,3,50,3,6)$ & $(0,25,0,65,1,05)$ & $(1.337,1.437,1.537)$ & $(2,3,4)$ & $(5,09,6,09,7,09)$ \\
MF-6 & $(0,17,0,27,0,37)$ & $(0,1,0,5,0,9)$ & $(281,381,481)$ & $(1,2,3)$ & $(3,55,4,55,5,55)$ \\
MF-7 & $(0,39,0,49,0,59)$ & $(0,41,0,81,1,21)$ & $(206,306,406)$ & $(1,2,3)$ & $(8,35,9,35,10,35)$ \\
\hline
\end{tabular}


$\tilde{y}_{r j}$ é o valor do limitante inferior no intervalo de definição da função de pertinência triangular da $r$-ésima saída Fuzzy para a DMU j , considerando a média o valor mais provável, sem incerteza.

$\widetilde{Y}_{r j}$ é o valor do limitante superior no intervalo de definição da função de pertinência triangular da $r$-ésima saída $F u z z y$ para a DMU $j$, considerando a média o valor mais provável, sem incerteza.

$\tilde{x}_{i j}$ é o valor do limitante inferior no intervalo de definição da função de pertinência triangular da $i$-ésima entrada Fuzzy para a DMU $j$, considerando a média o valor mais provável, sem incerteza.

$\tilde{X}_{i j}$ é o valor do limitante superior no intervalo de definição da função de pertinência triangular da $i$-ésima entrada Fuzzy para a DMU j , considerando a média o valor mais provável, sem incerteza.

$\alpha$ é valor escolhido para a abordagem nível- $\alpha$, com variação $\alpha \in[0,1]$.

$\psi_{i o}$ é o coeficiente de $\alpha$ nas restrições vinculado à $i$-ésima entrada Fuzzy da $\mathrm{DMU}_{0}$.

$\rho_{j 0}$ é coeficiente de $\alpha$ nas restrições vinculado à j-ésima saída Fuzzy da DMU .

$\mathrm{P}_{r j}$ é o coeficiente de $\alpha$ nas restrições vinculado à $r$-ésima saída Fuzzy da DMU j.

$\psi_{i j}$ é o coeficiente de $\alpha$ nas restrições vinculado à $i$-ésima entrada Fuzzy da DMU $j$.

\section{Variáveis auxiliares}

As variáveis auxiliares $\mathrm{d}^{-}, \mathrm{d}^{+}, d^{-}, d_{3 j}^{-}, d_{j}$ são os desvios associados às metas atribuídas a cada objetivo, conforme explicado na seção 2.

\section{Variáveis de decisão}

$u_{r}$ é o peso associado à $r$-ésima saída.

$v_{i}$ é o peso associado à $i$-ésima entrada.

Modelo FUZZY GPDEA-CCR para análise da eficiência das DMUs num cenário pessimista.

$$
\min a=\left(d_{1}^{-}+d_{1}^{+}+d_{2}^{-}+\sum_{j \in J} d_{3 j}^{-}+\sum_{j \in J} d_{j}\right)
$$

s.a.:

$$
\begin{gathered}
\sum_{i \in I} v_{i}\left(\tilde{x}_{i o}+\psi_{i 0} \cdot \alpha\right)+d_{1}^{-}-d_{1}^{+}=1 \\
\sum_{r \in R} u_{r}\left(\tilde{Y}_{r 0}-\rho_{r 0} \cdot \alpha\right)+d_{2}^{-}-d_{2}^{+}=1 \\
\sum_{r \in R} u_{r}\left(\tilde{Y}_{r j}-\rho_{r j} . \alpha\right)-\sum_{i \in I} v_{i}\left(\tilde{x}_{i j}+\psi_{i j} \cdot \alpha\right)+d_{j}=0, \quad \forall j \in J \\
M-d_{j}+d_{3 j}^{-}-d_{3 j}^{+}=0, \quad \forall j \in J \\
u_{r} \geq 0, \quad \forall r \in R \\
v_{i} \geq 0, \quad \forall i \in I
\end{gathered}
$$

$$
\begin{gathered}
d_{j} \geq 0, \quad \forall j \in J \\
d_{1}^{+}, d_{1}^{-}, d_{2}^{+}, d_{2}^{-} \geq 0, \quad d_{3 j}^{-}, d_{3 j}^{+} \geq 0,
\end{gathered}
$$

FUZZY GPDEA-CCR para análise da eficiência das DMUs num cenário otimista.

$$
\min a=\left(d_{1}^{-}+d_{1}^{+}+d_{2}^{-}+\sum_{j \in J} d_{3 j}^{-}+\sum_{j \in J} d_{j}\right)
$$

s.a.:

$$
\begin{gathered}
\sum_{i \in I} v_{i}\left(\tilde{X}_{i o}-\psi_{i 0} \cdot \alpha\right)+d_{1}^{-}-d_{1}^{+}=1 \\
\sum_{r \in R} u_{r}\left(\tilde{y}_{r 0}+\rho_{r 0} \cdot \alpha\right)+d_{2}^{-}-d_{2}^{+}=1 \\
\sum_{r \in R} u_{r}\left(\tilde{y}_{r j}+\rho_{r j} \cdot \alpha\right)-\sum_{i \in I} v_{i}\left(\tilde{X}_{i j}-\psi_{i j} \cdot \alpha\right)+d_{j}=0, \quad \forall j \in J \\
M-d_{j}+d_{3 j}^{-}-d_{3 j}^{+}=0, \quad \forall j \in J \\
u_{r} \geq 0, \quad \forall r \in R \\
v_{i} \geq 0, \quad \forall i \in I \\
d_{j} \geq 0, \quad \forall j \in J \\
d_{1}^{+}, d_{1}^{-}, d_{2}^{+}, d_{2}^{-} \geq 0, \quad d_{3 j}^{-}, d_{3 j}^{+} \geq 0
\end{gathered}
$$

Para formular os modelos FUZZY GPDEA-BCC pessimista e FUZZY GPDEA-BCC otimista basta incluir a variável auxiliar irrestrita $c_{\mathrm{o}}$ de forma aditiva nas Equações 43 e 44 e 52 e 53, respectivamente.

Observe-se, ainda, que quando $\alpha=1$ tem-se a formulação dos modelos GPDEA-CCR e GPDEA-BCC desenvolvidos por Bal, Örkcü e Çelebioğlu (2010), que são aplicáveis em situações sem incerteza. Esses modelos FGPDEA foram aplicados ao problema de encontrar as eficiências das minifábricas com os dados das Tabelas 1 e 2 . A Tabela 3 mostra os resultados (que foram agregados em função da limitação de espaço no artigo) obtidos com a aplicação dos modelos FGPDEA-CCR.

Na Tabela 3, nas colunas 2 a 8, entre parênteses estão dispostos para o cenário pessimista e para o cenário otimista, nessa ordem, os valores de eficiência de cada DMU.

Cabe mencionar que quando o valor de $\alpha=0$ tem-se um cenário no qual o valor dos parâmetros que estão abaixo da média (dados sem incerteza) serão exatamente o valor do limite inferior da função de pertinência, e o valor dos parâmetros que estão acima 
da média (dados sem incerteza) serão exatamente os valores do limite superior da função de pertinência. Entretanto, quando $\alpha=1$ tem-se um cenário sem incerteza, isto é, um cenário no qual os valores dos parâmetros de saída e entrada são formados pelos dados médios da função de pertinência, sendo esses os valores mais prováveis ou sem incerteza.

Percebe-se pela análise da Tabela 3 que apenas a DMU 1 foi eficiente em todas as otimizações. A DMU 5 apresentou a pior eficiência média, com apenas $3,31 \%$, e a maior amplitude de variação, com $1,52 \%$ (Tabela 4), podendo, assim, ser considerada a DMU mais sensível à incerteza. Além disso, pelo índice $I_{i}$, essa DMU ficou na última posição no ranking de eficiência. Já a DMU 6 foi a segunda DMU menos sensível à incerteza, apresentando uma amplitude de variação de apenas $0,29 \%$ e, pelo índice $I_{j}$, ficou na segunda posição do ranking de eficiência. Também se pode observar que, na aplicação dos modelos FGPDEA-CCR, apenas a DMU 1 foi eficiente, tendo as demais DMUs apresentado um alto nível de ineficiência. Nesse contexto, apenas a DMU 6 obteve uma eficiência média superior a $50 \%$, atingindo o valor $51,33 \%$.

Pelo fato de se analisar diferentes minifábricas que utilizam diferentes tecnologias de fabricação, foram aplicados também os modelos FGPDEA-BCC (com Retorno Variável de Escala) - os resultados estão nas Tabelas 5 e 6. Na Tabela 5, nas colunas 2 a 8, entre parênteses estão dispostos para o cenário pessimista e para o cenário otimista, nessa ordem, os valores de eficiência de cada DMU.
Analisando-se os resultados nas Tabelas 5 e 6, constata-se que o modelo FGPDEA-BCC também indicou que apenas a DMU 1 foi eficiente em todas as otimizações realizadas com variações do nível- $\alpha$. Já a DMU 3 apresentou o pior valor de eficiência média (31,60\%), ficando na última posição no ranking de eficiência medido pelo valor de $I_{j}$. Outra constatação foi que a DMU 4 apresentou a maior sensibilidade à incerteza, com um valor de amplitude de 75,88\%, ficando na penúltima posição do ranking de eficiência.

Adicionalmente, a aplicação dos modelos FGPDEA-BCC permitiu a observação de que, para os valores de nível- $\alpha$ com $\alpha=\{0,5 ; 0,6 ; 0,7 ; 0,8$; $0,9 ; 1\}$ há uma redução nos níveis de ineficiências das DMUs 2, 4, 6 e 7, ou seja, elas ficaram mais próximas de serem eficientes. Destaque-se a DMU 6 , que apresentou o menor nível de ineficiência, com um valor de $65,62 \%$, e a maior eficiência média, com um valor de $80,40 \%$.

Tais informações podem servir de alerta para os gestores das minifábricas, ou seja, eles devem investigar a razão de tal cenário, identificar os pontos falhos e reforçar os pontos fortes, tendo como objetivo a busca pela eficiência plena de todas as minifábricas.

De fato, os modelos FGPDEA-CCR e FGPDEA-BCC apontaram aos gestores os pontos críticos da atual administração das sete minifábricas e forneceram, também, a identificação das possibilidades de melhoria nas minifábricas ineficientes. Isso poderia ser operacionalizado, por exemplo, com a organização de reuniões que promovam a troca de experiências

Tabela 3. Cenários dos valores de eficiência (\%) em função do nível- $\alpha$ nos modelos FGPDEA-CCR.

\begin{tabular}{cccccccc}
\hline$\alpha$ & DMU 1 & DMU 2 & DMU 3 & DMU 4 & DMU 5 & DMU 6 & DMU 7 \\
\hline 0 & $(1,0,1,0)$ & $(0,178,0,244)$ & $(0,080,0,122)$ & $(0,027,0,100)$ & $(0,007,0,043)$ & $(0,492,0,534)$ & $(0,161,0,225)$ \\
0,1 & $(1,0,1,0)$ & $(0,182,0,231)$ & $(0,084,0,123)$ & $(0,031,0,096)$ & $(0,010,0,043)$ & $(0,494,0,532)$ & $(0,164,0,222)$ \\
0,2 & $(1,0,1,0)$ & $(0,186,0,228)$ & $(0,088,0,123)$ & $(0,035,0,093)$ & $(0,013,0,043)$ & $(0,496,0,530)$ & $(0,168,0,219)$ \\
0,3 & $(1,0,1,0)$ & $(0,189,0,225)$ & $(0,091,0,123)$ & $(0,039,0,089)$ & $(0,016,0,043)$ & $(0,498,0,528)$ & $(0,171,0,216)$ \\
0,4 & $(1,0,1,0)$ & $(0,192,0,222)$ & $(0,094,0,123)$ & $(0,042,0,086)$ & $(0,019,0,042)$ & $(0,500,0,527)$ & $(0,175,0,213)$ \\
0,5 & $(1,0,1,0)$ & $(0,196,0,218)$ & $(0,097,0,122)$ & $(0,046,0,082)$ & $(0,021,0,041)$ & $(0,502,0,525)$ & $(0,178,0,210)$ \\
0,6 & $(1,0,1,0)$ & $(0,199,0,215)$ & $(0,099,0,122)$ & $(0,050,0,079)$ & $(0,024,0,040)$ & $(0,504,0,523)$ & $(0,181,0,207)$ \\
0,7 & $(1,0,1,0)$ & $(0,202,0,212)$ & $(0,101,0,121)$ & $(0,054,0,075)$ & $(0,026,0,038)$ & $(0,506,0,521)$ & $(0,185,0,204)$ \\
0,8 & $(1,0,1,0)$ & $(0,205,0,209)$ & $(0,103,0,12)$ & $(0,058,0,071)$ & $(0,029,0,037)$ & $(0,508,0,519)$ & $(0,188,0,200)$ \\
0,9 & $(1,0,1,0)$ & $(0,208,0,206)$ & $(0,104,0,119)$ & $(0,062,0,067)$ & $(0,031,0,035)$ & $(0,510,0,517)$ & $(0,191,0,197)$ \\
1 & 1 & 0,203 & 0,105 & 0,064 & 0,033 & 0,515 & 0,194 \\
$\mathbf{I}_{j}$ & $\mathbf{1}$ & $\mathbf{0 , 2 0 8}$ & $\mathbf{0 , 1 1 1}$ & $\mathbf{0 , 0 7 2}$ & $\mathbf{0 , 0 3 2}$ & $\mathbf{0 , 5 1 0}$ & $\mathbf{0 , 1 9 7}$ \\
\hline
\end{tabular}

Tabela 4. Valores aglutinados dos cenários pessimista e otimista vinculados a eficiência gerada pelo modelo FGPDEA-CCR.

\begin{tabular}{lccccccc}
\hline Média & 100,00 & 20,62 & 10,69 & 6,02 & 2,81 & 51,33 & 19,29 \\
Máximo & 100,00 & 20,74 & 11,14 & 6,45 & 3,31 & 51,52 & 19,42 \\
Mínimo & 100,00 & 20,33 & 9,92 & 5,15 & 1,80 & 51,24 & 19,04 \\
Amplitude & 0,00 & 0,41 & 1,22 & 1,30 & 1,52 & 0,29 & 0,38 \\
\hline
\end{tabular}


Tabela 5. Cenários dos valores de eficiência (\%) em função do nível- $\alpha$ nos modelos FGPDEA-BCC.

\begin{tabular}{cccccccc}
\hline$\alpha$ & DMU 1 & DMU 2 & DMU 3 & DMU 4 & DMU 5 & DMU 6 & DMU 7 \\
\hline 0 & $(1,0,1,0)$ & $(0,162,0,801)$ & $(0,056,0,482)$ & $(0,015,0,767)$ & $(0,000,0,572)$ & $(0,490,0,879)$ & $(0,152,0,799)$ \\
0,1 & $(1,0,1,0)$ & $(0,162,0,801)$ & $(0,060,0,475)$ & $(0,015,0,767)$ & $(0,000,0,567)$ & $(0,490,0,879)$ & $(0,152,0,799)$ \\
0,2 & $(1,0,1,0)$ & $(0,162,0,801)$ & $(0,063,0,468)$ & $(0,015,0,767)$ & $(0,000,0,562)$ & $(0,490,0,879)$ & $(0,152,0,799)$ \\
0,3 & $(1,0,1,0)$ & $(0,162,0,801)$ & $(0,067,0,461)$ & $(0,015,0,767)$ & $(0,000,0,557)$ & $(0,490,0,879)$ & $(0,152,0,799)$ \\
0,4 & $(1,0,1,0)$ & $(0,162,0,801)$ & $(0,070,0,454)$ & $(0,015,0,767)$ & $(0,000,0,551)$ & $(0,490,0,879)$ & $(0,152,0,799)$ \\
0,5 & $(1,0,1,0)$ & $(0,882,0,801)$ & $(0,378,0,446)$ & $(0,861,0,767)$ & $(0,516,0,545)$ & $(0,928,0,879)$ & $(0,881,0,799)$ \\
0,6 & $(1,0,1,0)$ & $(0,980,0,801)$ & $(0,436,0,438)$ & $(0,977,0,767)$ & $(0,601,0,539)$ & $(0,988,0,879)$ & $(0,980,0,799)$ \\
0,7 & $(1,0,1,0)$ & $(0,980,0,801)$ & $(0,450,0,430)$ & $(0,977,0,767)$ & $(0,615,0,532)$ & $(0,988,0,879)$ & $(0,980,0,799)$ \\
0,8 & $(1,0,1,0)$ & $(0,980,0,801)$ & $(0,464,0,421)$ & $(0,977,0,767)$ & $(0,628,0,525)$ & $(0,988,0,879)$ & $(0,980,0,799)$ \\
0,9 & $(1,0,1,0)$ & $(0,980,0,801)$ & $(0,478,0,411)$ & $(0,977,0,767)$ & $(0,640,0,517)$ & $(0,988,0,879)$ & $(0,980,0,799)$ \\
1 & 1,0 & 0,886 & 0,490 & 0,865 & 0,575 & 0,932 & 0,841 \\
$\mathbf{I}_{j}$ & $\mathbf{1 , 0}$ & $\mathbf{0 , 6 6 7}$ & $\mathbf{0 , 3 8 0}$ & $\mathbf{0 , 6 1 9}$ & $\mathbf{0 , 6 2 3}$ & $\mathbf{0 , 7 8 3}$ & $\mathbf{0 , 6 6 3}$ \\
\hline
\end{tabular}

Tabela 6. Valores aglutinados dos cenários pessimista e otimista vinculados a eficiência gerada pelo modelo FGPDEA-BCC.

\begin{tabular}{lccccccc}
\hline Média & 100,00 & 64,28 & 31,60 & 51,55 & 56,30 & 80,40 & 63,68 \\
Máximo & 100,00 & 88,62 & 44,36 & 86,52 & 57,54 & 93,19 & 88,48 \\
Mínimo & 100,00 & 35,99 & 16,49 & 10,64 & 0,02 & 65,62 & 34,84 \\
Amplitude & 0,00 & 52,64 & 27,88 & 75,88 & 57,52 & 27,57 & 53,65 \\
\hline
\end{tabular}

entre os gestores de cada minifábrica a partir dos resultados apontados pelos modelos.

Cabe mencionar que o modelo Fuzzy GPDEA é uma alternativa robusta para avaliar a eficiência quando a Regra de Ouro (Golden Rule) estabelecida por Banker et al. (1989) para o uso de modelos clássicos da DEA não é atendida. Quando essa regra não é atendida, os modelos clássicos (CCR e BCC) não proporcionam uma discriminação confiável entre as DMUs (BANKER et al., 1989). A Regra de Ouro recomenda que, para utilizar os modelos da DEA (CCR e BCC), o número de DMUs deve ser pelo menos igual a três vezes a soma total do número de variáveis (inputs e outputs) envolvidas, ou pelo menos igual ao produto do número de variáveis de input e output, adotando-se o critério associado ao maior número de DMUs necessárias.

\section{Conclusões e recomendações para futuras pesquisas}

Muitos estudos com modelos DEA tratam casos determinísticos, ou seja, todas as variáveis de entrada e de saída podem ser medidas com precisão e com boa acuracidade, mas os problemas reais são naturalmente incertos ou imprecisos, ou seja, nem sempre se tem a qualidade desejada nas informações disponíveis.

A contribuição de mais destaque deste trabalho foi a proposição de modelos FGPDEA-CCR e FGPDEA-BCC para avaliar a eficiência de DMUs num cenário sob incerteza onde a Regra de Ouro, formulada por Banker et al. (1989), não é atendida.
Do ponto de vista gerencial, esses modelos podem proporcionar uma análise de eficiência mais robusta, além de promover uma maior interação entre os gestores, com vistas a um melhor entendimento do problema tratado, notadamente na tarefa de estabelecer os intervalos de variação (range da incerteza) das variáveis envolvidas.

De fato, foi consenso entre os gestores consultados, pertencentes à empresa objeto do estudo, que os modelos FGPDEA permitiram uma maior interação entre os gestores e os analistas, fazendo com que o desenvolvimento, a otimização e a análise dos resultados dos modelos fossem aceitos mais facilmente pelo corpo gerencial da empresa, o que vai facilitar a implementação de propostas advindas dos resultados obtidos com os modelos GPDEA já comentados.

Vale a pena comentar que, apesar de a etapa de implementação fugir do escopo deste trabalho, os gestores já decidiram desenvolver políticas para minimizar as incertezas nas DMUs e desenvolver estratégias visando a melhor alocação dos recursos e aumento da produtividade das minifábricas, tendo como base os resultados dos modelos FGPDEA-BCC da seção 4 e como benchmark a DMU 1.

Destaque-se, ainda, que a combinação da lógica Fuzzy com o modelo GPDEA não agrega maiores complexidades computacionais, como atesta o tempo gasto para otimizar os 11 cenários considerados, que foi de aproximadamente dez minutos. Assim, a nova estrutura algébrica de modelos GPDEA, que é 
de fácil implementação computacional, ampliou o potencial de aplicações desses modelos.

Como recomendações para futuras pesquisas pretende-se combinar a Teoria dos Jogos da Barganha de Nash (SANTOS; MARINS; SALOMON, 2011) nos modelos GPDEA, visando possibilitar a inserção de estratégias de negociação entre os clientes e produtores em um ambiente sob incerteza.

\section{Agradecimentos}

Ao CNPq e à Capes, pelo apoio, bem como aos revisores anônimos que fizeram sugestões interessantes para a melhoria do texto.

\section{Referências}

AOUNI, B.; MARTEL, J-M.; HASSAINE, A. Fuzzy Goal Programming Model: An Overview of the Current State-of-the Art. Journal of Multi-Criteria Decision Analysis, v. 16, n. 5-6, p. 149-161, 2009. http://dx.doi. org/10.1002/mcda.448

BAL, H; ÖRKCÜ, H. H.; ÇELEBIOĞLU, S. Improving the discrimination power and weights dispersion in the data envelopment analysis. Computers \& Industrial Engineering, v 37, n. 1, p. 99-107, 2010.

BANKER, R. D.; CHARNES, A.; COOPER, W. W. Some models for estimating technical and scale inefficiencies in Data Envelopment Analysis. Management Science, v. 30, n. 9, p. 1078-1092, 1984. http://dx.doi.org/10.1287/ mnsc.30.9.1078

BANKER, R. D. et al. An Introduction to Data Envelopment Analysis with Some of its Models and Their Uses. Research in Governmental and Non-Profit Accounting, v. 5, p. 125-163, 1989.

BERTRAND, J. W. M.; FRANSOO, J. C. Operations management research methodologies using quantitative modeling. International Journal of Operations and Production Management, v. 22, p. 241-264, 2002. http://dx.doi.org/10.1108/01443570210414338

COOK, W. D.; SEIFORD, L. M. Data Envelopment Analysis (DEA) - Thirty years on. European Journal of Operational Research, v. 192, p. 1-17, 2009. http:// dx.doi.org/10.1016/j.ejor.2008.01.032

COOPER, W. W.; SEIFORD, L. M.; TONE, K. Introduction to Data Envelopment Analysis and its uses: with DEA-Solver software and references. New York: Springer, 2006.

COOPER, W. W.; PARK, K. S.; YU, G. IDEA and AR-IDEA: Models for dealing with imprecise data in DEA. Management Science, v. 45, p. 597-607, 1999. http://dx.doi.org/10.1287/mnsc.45.4.597

CHARNES, A.; COOPER, W. W. Management Models and Industrial Applications of Linear Programming. Wiley: New York, 1961.

CHARNES, A.; COOPER, W. W. Programming with linear fractional functional. Naval Research Logistics Quarterly, v. 9, n. 3-4, p. 181-185, 1962. http://dx.doi. org/10.1002/nav.3800090303

CHARNES, A.; COOPER, W. W.; RHODES, E. Measuring the efficiency of decision making units. European Journal of Operational Research, v. 2, n. 6, p. 429-444, 1978. http://dx.doi.org/10.1016/0377-2217(78)90138-8

CHEN, C. B.; KLEIN, C. M. A simple approach to ranking a group of aggregated fuzzy utilities. IEEE Transactions on Systems, Man, and Cybernetics, Part B: Cybernetics, v. 27, p. 26-35, 1997. PMid:18255837. http://dx.doi.org/10.1109/3477.552183

ENTANI, T.; MAEDA, Y.; TANAKA, H. Dual models of interval DEA and its extension to interval data. European Journal of Operational Research, v. 136, p. 32-45, 2002. http://dx.doi.org/10.1016/ S0377-2217(01)00055-8

FIGUEIREDO, D. S.; MELLO, J. C. C. B. S. Índice híbrido de eficácia e eficiência para lojas de varejo. Gestão $\boldsymbol{\&}$ Produção, v. 16, n. 2, p. 286-300, 2009. http://dx.doi. org/10.1590/S0104-530X2009000200011

GARCIA, P. A. A.; MELO, P. F. F.; SCHIRRU, R. Aplicação de um modelo Fuzzy DEA para priorizar modos de falhas em sistemas nucleares. Pesquisa Operacional, v. 29, p. 383-402, 2009. http://dx.doi.org/10.1590/ S0101-74382009000200007

GUO, P.; TANAKA, H. Fuzzy DEA: A perceptual evaluation method. Fuzzy Sets and Systems, v. 119, p. 149-160, 2001. http://dx.doi.org/10.1016/ S0165-0114(99)00106-2

HATAMI-MARBINI, A.; EMROUZNEJAD, A.; TAVANA, M. A. Taxonomy and review of the Fuzzy data Envelopment Analysis literature: Two decades in the making. European Journal of Operational Research, v. 214, p. 457-472, 2011. http://dx.doi.org/10.1016/j. ejor.2011.02.001

KAO, C.; LIU, S. T. Fuzzy efficiency measures in data envelopments analysis. Fuzzy Sets and Systems, v. 133 , p. 427-437, 2000. http://dx.doi.org/10.1016/ S0165-0114(98)00137-7

KAO, C.; LIN, P. H. Efficiency of parallel production systems with Fuzzy data. Fuzzy Sets and Systems, v. 19, p. 83-98, 2012. http://dx.doi.org/10.1016/j. fss.2012.01.004

KAUFMANN, A. Introduction to the Theory of Fuzzy Subsets. New York: Academic Press, 1975. v. 1. PMid:13983.

LI, X-B.; REEVES, G. R. A multiple criteria approach to data envelopment analysis. European Journal of Operational Research, v. 115, n. 3, p. 507-517, 1999. http://dx.doi.org/10.1016/S0377-2217(98)00130-1

LIANG, G-S.; WANG, M-J. Evaluating Human Reliability Using Fuzzy Relation. Microelectronics Reliability, v. 33, n. 1, p. 63-80, 1993. http://dx.doi. org/10.1016/0026-2714(93)90046-2

LERTWORASIRIKUL, S. et al. Fuzzy data envelopment analysis (DEA): A possibility approach. Fuzzy Sets and Systems, v. 139, p. 379-394, 2003. http://dx.doi. org/10.1016/S0165-0114(02)00484-0

SANTOS, M. A.; MARINS, F. A. S.; SALOMON, V. A. P. Utilização da Função Arbitragem de Nash nos Modelos DEA CCR: Uma abordagem na orientação combinada entrada/saída sob a ótica da Teoria dos Jogos de Barganha. In: SIMPÓSIO BRASILEIRO DE PESQUISA OPERACIONAL - SBPO, 44., 2012. Rio de Janeiro. Anais... SOBRAPO, 2012. 
SILVA, A. F.; MARINS, F. A. S.; MONTEVECHI, J.

A. B. Multi-choice mixed integer goal programming optimization for real problems in a sugar and ethanol milling company. Applied Mathematical Modelling, v. 37, p. 6146- 6162, 2013a. http://dx.doi.org/10.1016/j. apm.2012.12.022

SILVA, A. F.; MARINS, F. A. S.; MONTEVECHI, J. A. B. Aplicação de programação por metas binária mista em uma empresa do setor sucroenergético. Gestão \& Produção, v. 20, p. 321-336, 2013b. http:// dx.doi.org/10.1590/S0104-530X2013000200006

WEN, M.; QIN, Z.; KANG, R. Sensitivity and stability analysis in Fuzzy data envelopment analysis. Fuzzy Optimization Decision Making, v. 10, p. 1-10, 2011. http://dx.doi.org/10.1007/s10700-010-9093-y
WEN, M.; YOU, C. A. Fuzzy data envelopment analysis (DEA) model with credibility measure. Technical Report, 2007.

YAGER, R. R. A characterization of the extension principle. Fuzzy Sets and Systems, v. 18, p. 205-217, 1986. http:// dx.doi.org/10.1016/0165-0114(86)90002-3

ZADEH, L. A. Outline of a new approach to the analysis of complex systems and decision processes. IEEE Trans. Systems Man Cybernet, v. 1, p. 28-44, 1973. http:// dx.doi.org/10.1109/TSMC.1973.5408575

ZADEH, L. A. Fuzzy sets as a basis for a theory of possibility. Fuzzy Sets and Systems, v. 1, p. 3-28, 1978. http:// dx.doi.org/10.1016/0165-0114(78)90029-5

ZIMMERMANN, H. J. Fuzzy Set Theory and Its Applications. 2nd ed. Boston: Kluwer-Nijhoff, 1991. http://dx.doi.org/10.1007/978-94-015-7949-0
Anexo A. Matriz de outputs e inputs das sete minifábricas. Tabela 7. Matriz de outputs.

\begin{tabular}{ccc}
\hline DMU & $\mathbf{Y}_{1}$ & $\mathbf{Y}_{2}$ \\
\hline MF-1 & 2.510 & 2.494 \\
MF-2 & 531 & 507 \\
MF-3 & 592 & 525 \\
MF-4 & 164 & 159 \\
MF-5 & 124 & 124 \\
MF-6 & 1.285 & 1.285 \\
MF-7 & 488 & 484 \\
\hline
\end{tabular}

Tabela 8. Matriz de inputs.

\begin{tabular}{cccccc}
\hline DMU & $\mathbf{X}_{1}$ & $\mathbf{X}_{2}$ & $\mathbf{X}_{3}$ & $\mathbf{X}_{4}$ & $\mathbf{X}_{5}$ \\
\hline MF-1 & 0,16 & 0,42 & 0 & 2 & 4,83 \\
MF-2 & 0,69 & 0,92 & 111 & 2 & 11,30 \\
MF-3 & 0,51 & 0,54 & 398 & 4 & 1,23 \\
MF-4 & 2,31 & 0,56 & 194 & 2 & 15,47 \\
MF-5 & 3,50 & 0,65 & 1.437 & 3 & 6,09 \\
MF-6 & 0,27 & 0,50 & 381 & 2 & 4,55 \\
MF-7 & 0,49 & 0,81 & 306 & 2 & 9,35 \\
\hline
\end{tabular}

